\section{IMPACTO DO TABAGISMO NA SAÚDE BUCAL DOS PACIENTES ATENDIDOS EM UM AMBULATÓRIO DE CLÍNICA MÉDICA}

\author{
Impact of smoking on oral health of patients attending an \\ outpatient medical clinic
}

\author{
Impacto del tabaco en la salud bucal de pacientes asistidos en \\ ambulatório de clínica médica
}

\section{RESUMO}

Objetivo: Avaliar o impacto do tabagismo na saúde bucal dos pacientes atendidos em um ambulatório de clínica médica de um hospital de referência. Métodos: Realizou-se um estudo transversal no período de setembro de 2011 a julho de 2012 com 125 pacientes, os quais foram avaliados através de questionários quanto aos dados sociodemográficos, qualidade da saúde bucal e hábito tabagístico. Analisaram-se os dados através da correlação de Pearson e teste de regressão logística, com $p<0,05$. Resultados: Os pacientes tinham idade média de 50 $\pm 15,5$ anos e $52 \%$ (65) eram do sexo feminino. Quanto ao hábito tabagístico 28,8\% (36) eram fumantes, 28,8\% (36) ex-fumantes e 42,4\% (53) nunca fumaram. Observou-se que 53,3\% (67) dos pacientes perderam mais de 5 dentes nos últimos 10 anos, 55,2\% (69) apresentavam alteração na cor dos dentes, 40\% (50) relataram halitose e 54\% (68) apresentavam ulcerações ou manchas na boca. A perda dos dentes foi mais frequente entre os fumantes atuais $(86 \%)$ quando comparado aos ex-fumantes $(63 \%)$ e não fumantes $(24 \%)$. Encontrou-se associação do tabagismo com perda de dentes (OR 2,7; IC95\% 1,09-6,93) e alteração na cor dos dentes (OR 2,5; IC95\% 1,03-6,41). Conclusão: Na amostra estudada, o tabagismo está associado à deterioração da saúde bucal, causando, pois, um impacto negativo sobre esta.

Descritores: Hábito de Fumar; Saúde Bucal; Fatores de Risco.

\section{ABSTRACT}

Objective: To evaluate the impact of smoking on the oral health of patients attending the outpatient medical clinic of a reference hospital. Methods: A cross-sectional study was conducted in the period of September 2011 to July 2012 with 125 patients attending the ambulatory of the Federal University of Ceará. Patients were assessed through questionnaires regarding socio-demographic data, oral health quality and smoking habits. Data was analyzed using logistic regression and Pearson test, with $p<0.05$. Results: Patients' mean age was $50 \pm 15.5$ years and $52 \%$ (65) of them were female. As for the smoking habits, $28.8 \%$ (36) were current smokers, $28.8 \%$ (36) former smokers and $42.4 \%$ (53) non-smokers. It was observed that $53.3 \%$ (67) of the patients had lost more than five teeth in the last 10 years, $55.2 \%$ (69) had a change in the teeth color, 40\% (50) reported halitosis and 54\% (68) had ulcers or spots in the mouth. Teeth loss was more common for current smokers (86\%) than among former smokers (63\%) and non-smokers (24\%). Smoking was found to be associated with tooth loss (OR 2.7; 95\% CI 1.09-6.93) and with change in the teeth color (OR 2.5; 95\% CI 1.03-6.41). Conclusion: In the studied sample, smoking is associated with deterioration of oral health, thus causing on it a negative impact.

Descriptors: Smoking; Oral Health; Risk Factors.
Artigo Original

Eanes Delgado Barros Pereira ${ }^{(1)}$ Edyr Pereira Paiva Freitas ${ }^{(1)}$ Bianca Almeida Moreira ${ }^{(1)}$ Felipe Augusto de Paula ${ }^{(1)}$ Renata Delgado Pereira dos Santos $^{(2)}$

Antonio George Cavalcante Matos $^{(3)}$

1) Universidade Federal do Ceará - UFC Fortaleza (CE) - Brasil

2) Universidade de Fortaleza - UNIFOR Fortaleza (CE) - Brasil

3) Hospital Universitário Walter Cantídio HUWC - Fortaleza (CE) - Brasil

Recebido em: 04/01/2103

Revisado em: 27/08/2013 Aceito em: 12/12/2013 


\section{RESUMEN}

Objetivo: Evaluar el impacto del tabaco en la salud bucal de pacientes asistidos en un ambulatorio de clínica médica en un hospital de referencia. Métodos: Se realizó un estudio trasversal entre septiembre de 2011 y julio de 2012 con 125 pacientes los cuales fueron evaluados con cuestionarios sobre datos sociodemográficos, calidad de salud bucal y el hábito del tabaco. Se analizó los datos a través de la correlación de Pearson y el test de regresión logístico con $p<0,05$. Resultados: Los pacientes presentaron edad media de $50 \pm 15,5$ años y el 52\% (65) era del sexo femenino. Sobre el hábito del tabaco, el 28,8\% (36) era fumadores, el 28,8\% (36) ex-fumadores y el 42,4\% (53) nunca había fumado. Se observó que el 53,3\% (67) de los pacientes perdieron más de 5 dientes en los últimos 10 años, el 55,2\% (69) presentó alteración del color de los dientes, el $40 \%$ (50) relató halitosis y el 54\% (68) presentó ulceraciones o manchas en la boca. La pérdida de los dientes fue más frecuente en los fumadores actuales (86\%) al comparar a los ex-fumadores (63\%) y no fumadores (24\%). Se encontró una asociación del tabaquismo y la pérdida de los dientes (OR 2,7; IC 95\% 1,09-6,93) y alteración del color de los dientes (OR 2,5; IC95\% 1,03-6,41). Conclusión: El tabaquismo está asociado a la deterioración de la salud bucal en la muestra estudiada, llevando, así, a un impacto negativo sobre ella.

Descriptores: Hábito de fumar; Salud bucal; Factores de Riesgo.

\section{INTRODUÇÃO}

O tabagismo é um dos mais importantes problemas de saúde pública e uma das principais causas preveníveis de morte no mundo ${ }^{(1)}$. Estima-se que, caso nenhuma ação seja realizada, o número anual de óbitos causados pelo uso do tabaco nos países desenvolvidos atingirá 7 milhões no ano $2020^{(2)}$ No Brasil, um terço da população adulta fuma, de acordo com dados do Instituto Nacional de Câncer (INCA), e o número estimado de óbitos relacionados ao fumo para 2014 é de 580.000 casos $^{(3)}$.

Além de ser prejudicial à saúde geral, o tabagismo também compromete a saúde bucal ${ }^{(4,5)}$. O hábito de fumar foi associado ao aumento de risco de câncer bucal, leucoplasia, gengivite ulcerativa necrosante aguda, candidíase bucal, insucesso de implantes dentais e doença periodontal, além de interferir no resultado de terapias periodontais cirúrgicas e não cirúrgicas ${ }^{(4-9)}$. Fumar resulta em vasoconstrição periférica e, consequentemente, em prejuízo à cicatrização de feridas na boca ${ }^{(6)}$. Causa também descoloração nos dentes e nas restaurações dentais, prejudica os sentidos do olfato e paladar, e frequentemente provoca halitose $\mathrm{e}^{(6-9)}$.

$\mathrm{O}$ hábito de mascar tabaco geralmente causa enrugamento da mucosa bucal, recessão gengival e câncer na cavidade oral ${ }^{(6)}$. Estudos epidemiológicos revelaram uma maior prevalência e gravidade da doença periodontal em fumantes quando comparados aos não fumantes, indicando a ação direta do tabaco nos tecidos da cavidade oral ${ }^{(4,6,8)}$.

Em virtude disso, destaca-se o papel do dentista e do médico em alertar os pacientes para os riscos à saúde causados pelo fumo, incluindo não somente o risco de doença periodontal, mas de câncer oral ${ }^{(5,7-10)}$. Os estudos que envolvem tabagismo e comprometimento da saúde bucal em geral são realizados com especialistas em clínicas odontológicas ou na comunidade ${ }^{(11-15)}$. Assim, torna-se importante realizar estudos com populações específicas, como pacientes acompanhados pelo clínico geral. Desta forma, estes profissionais serão alertados acerca dos problemas que envolvem a saúde bucal de seus pacientes, encaminhando-os mais precocemente para um atendimento odontológico especializado.

O objetivo do estudo foi avaliar o impacto do tabagismo na saúde bucal dos pacientes atendidos em um ambulatório de clínica médica de um hospital de referência.

\section{MÉTODOS}

Realizou-se um estudo caso-controle no Ambulatório de Clínica Médica do Hospital Universitário Walter Cantídio, da Universidade Federal do Ceará, no período de setembro de 2011 a julho de 2012.

Selecionaram-se consecutivamente pacientes que compareceram ao ambulatório de clínica médica para consulta regular através de amostragem não probabilística e por conveniência. Todos tinham idade acima de 18 anos e estavam em acompanhamento ambulatorial há mais de seis meses.

Por meio de um questionário elaborado pelos autores, realizou-se a coleta dos seguintes dados: dados demográficos (idade e sexo), hábito tabagístico, qualidade da saúde bucal, hábitos alimentares (comer alimentos com excesso de açúcar pelo menos cinco vezes por semana), etilismo e higienização da boca. A aplicação do questionário ocorria no momento em que os pacientes compareciam para consulta. Inicialmente, realizou-se um estudo piloto com 10 pacientes para esclarecimento de dúvidas quanto às perguntas. Houve um treinamento prévio dos membros da equipe para aplicação do questionário para minimizar os erros de coleta.

A definição do hábito tabagístico seguiu os critérios da Organização Mundial da Saúde ${ }^{(16)}$ : fumante regular, aqueles indivíduos que haviam fumado um mínimo de um cigarro por dia por pelo menos 6 meses antes do início do estudo; ex-fumante, aqueles que haviam fumado e parado de fumar há pelo menos 6 meses antes do início do estudo; 
e não-fumante, aqueles que nunca fumaram. A definição de etilismo seguiu os critérios da Organização Mundial da Saúde ${ }^{(17)}$ : beber diariamente mais de duas latas de cerveja ou duas doses de destilado, como uísque ou cachaça.

Considerou-se má qualidade da saúde bucal nos pacientes que apresentavam um dos seguintes critérios adaptados $^{(11-15)}$ : perda de mais de cinco dentes nos últimos 10 anos, presença de descoloração dos dentes, presença de ulcerações ou manchas na boca. A higienização da boca foi baseada na recomendação da Associação Americana de Cirurgiões-Dentistas ${ }^{(18)}$.

As variáveis demográficas, clínicas e da saúde bucal foram apresentadas em tabelas através de proporções. Fatores associados à má qualidade da saúde bucal receberam análise pelo teste do qui-quadrado de Pearson. Aplicou-se um modelo de regressão logística multivariada para analisar os possíveis fatores de risco relacionados às condições da saúde bucal dos pacientes, tendo como variável independente a perda maior que cinco dentes nos últimos 10 anos, e como dependente, a alteração na cor dos dentes. Os fatores de risco incluídos no modelo multivariado foram sexo, idade, hábitos dietéticos, higienização da boca e etilismo, por mostrarem um nível de significância maior que $20 \%$ na análise univariada ou pela sua importância clínica. Considerou-se significativo o valor descritivo abaixo de $5 \%(\mathrm{p}<0,05)$, Odds ratio $(\mathrm{OR})$ ajustado e intervalo de confiança 95\% (IC 95\%), utilizando- se o programa estatístico Statistical Package for the Social Sciences, versão 16.0 (SPSS Inc., Chicago, IL, EUA).

O protocolo do estudo recebeu aprovação do comitê de ética em pesquisa da instituição com o número 041.04.11 em 09/05/2011. Todos os participantes assinaram o termo de consentimento livre e esclarecido.

\section{RESULTADOS}

Avaliaram-se 125 pacientes com idade média de 50,0 \pm 15,5 anos. A estratificação por faixa etária mostrou que $34 \%$ $(\mathrm{n}=43)$ estavam com idade igual ou superior a 60 anos; $41 \%$ $(n=52)$ entre 40 e 59 anos; e $24 \%(n=30)$ igual ou inferior a 39 anos. Entre estes pacientes, $52 \%(n=65)$ eram do sexo feminino.

Quanto ao hábito tabagístico, $28,8 \%(\mathrm{n}=36)$ eram fumantes, $28,8 \%(n=36)$ ex-fumantes e $42,4 \%(n=53)$ não fumantes.

Entre os pacientes, $96 \%(\mathrm{n}=121)$ relataram que faziam higienização da boca, $52,8 \%(\mathrm{n}=66)$ relataram comer alimentos com excesso de açúcar e 14,4\% ( $n=18)$ faziam ingestão de álcool regularmente. Quanto às alterações da saúde bucal, foi observado que $53,3 \%(n=67)$ dos pacientes perderam mais de cinco dentes nos últimos 10 anos, 55,2\% $(n=69)$ apresentavam alteração na cor dos dentes, $40 \%$ $(n=50)$ relataram halitose e $54 \%(n=68)$ apresentavam ulcerações ou manchas na boca (Tabela I).

Tabela I - Características sociodemográficas, clínicas e da saúde bucal dos 125 pacientes atendidos no ambulatório de clínica médica do Hospital Universitário Walter Cantídio. Fortaleza-Ce, 2012.

\begin{tabular}{|c|c|c|}
\hline Variável & $\mathbf{n}$ & $\%$ \\
\hline \multicolumn{3}{|l|}{ Sexo } \\
\hline Feminino & 65 & 52,0 \\
\hline Masculino & 60 & 48,0 \\
\hline \multicolumn{3}{|l|}{ Faixa etária } \\
\hline$\geq$ a 60 anos & 43 & 34,0 \\
\hline$\overline{40}-59$ anos & 52 & 41,0 \\
\hline$<$ a 39 anos & 30 & 24,0 \\
\hline Higienização da boca & 121 & 96,8 \\
\hline Etilista & 18 & 14,4 \\
\hline \multicolumn{3}{|l|}{ Hábito tabagístico } \\
\hline Fumante atual & 36 & 28,9 \\
\hline Ex-fumante & 36 & 28,9 \\
\hline Não fumante & 53 & 42,4 \\
\hline \multicolumn{3}{|l|}{ Hábitos alimentares } \\
\hline Dieta com muito açúcar & 66 & 52,8 \\
\hline \multicolumn{3}{|l|}{ Qualidade da saúde bucal } \\
\hline Perda de mais de 5 dentes nos últimos 10 anos & 67 & 53,6 \\
\hline Ulceras/Manchas na boca & 68 & 54,0 \\
\hline Halitose & 50 & 40,0 \\
\hline Descoloração dos dentes & 69 & 55,0 \\
\hline
\end{tabular}


Houve uma associação significante entre o hábito tabagístico e a qualidade da saúde bucal dos pacientes. Os fumantes apresentaram um maior percentual de perda de dentes e alteração na cor dos dentes em relação aos exfumantes e não fumantes (Tabela II).
Pelo processo backward stepwise, as variáveis que apresentaram uma associação com a perda maior que 5 dentes nos últimos 10 foram tabagismo $(\mathrm{OR}=2,7$; IC $95 \% 1,09-6,93)$ e idade maior que 60 anos $(\mathrm{OR}=2,7$; IC $95 \%$ 1,25-6,13). Para avaliar a alteração na cor dos dentes, observou-se que o tabagismo apresentou uma associação significante $(\mathrm{OR}=2,5$; IC95\% 1,03-6,41) (Tabela III).

Tabela II - Associação entre o hábito tabagístico e alterações bucais de 125 pacientes atendidos no ambulatório de clínica médica do Hospital Universitário Walter Cantídio. Fortaleza-Ce, 2012.

\begin{tabular}{lccc}
\hline Variável & Fumante atual & Ex-fumante & Não Fumante \\
& $\mathbf{( 3 6 )}$ & $\mathbf{( 3 6 )}$ & $\mathbf{( 5 3 )}$ \\
\hline Perda de mais de 5 dentes nos últimos 10 anos (\%) & $86 \%^{*}$ & $63 \% * *$ & $24 \%$ \\
Descoloração dos dentes (\%) & $72 \% \dagger+$ & $44 \%$ & $50 \%$ \\
Ulceras/manchas na boca (\%) & $11 \%$ & $2 \%$ & $3,9 \%$ \\
\hline
\end{tabular}

*Diferença em relação aos não fumantes $(\mathrm{p}=0,001),{ }^{* *}$ Diferença em relação aos não fumantes, $\dagger$ Diferença em relação aos ex-fumantes $(p=0,03)$, \$Diferença em relação aos não fumantes $(p=0,04)$. Utilizado o teste do qui-quadrado de Pearson

Tabela III - Análise de regressão logística para avaliar associação do tabagismo com saúde bucal controlando para sexo, idade, história de alcoolismo, hábitos dietéticos, higienização da boca. Fortaleza-Ce, 2012.

\begin{tabular}{lccc}
\hline Desfecho/preditor & Odds Ratio ajustado & IC* 95\% & $\boldsymbol{p}$ \\
\hline Perda de dentes & & & 0,01 \\
Fumante atual & 2,7 & $1,09-6,93$ & 0,03 \\
Idade $\geq 60$ anos & 2,7 & $1,25-6,13$ & \\
Alteração da cor dos dentes & 2,5 & $1,03-6,41$ & 0,04 \\
Fumante atual & & \\
\hline
\end{tabular}

*IC = Intervalo de Confiança

\section{DISCUSSÃO}

O presente estudo mostrou que o tabagismo é um fator de risco para perda dos dentes e alteração na cor dos dentes entre os pacientes atendidos no ambulatório de clínica médica de um hospital de ensino.

Demonstra-se a associação do fumo com a má qualidade da saúde bucal, sendo o hábito de fumar um dos principais fatores de risco para o desenvolvimento e progressão da doença periodontal ${ }^{(8-10,13)}$. Esta condição é caracterizada por processo inflamatório crônico da gengiva e/ou dos tecidos de suporte dos dentes, o que pode ocasionar reabsorção óssea alveolar, aumento da mobilidade dental, exposição das raízes e perda dos dentes ${ }^{(15)}$.

No presente estudo, um percentual de $86 \%$ dos participantes relataram perda superior a 5 dentes nos últimos 10 anos. Este resultado foi superior ao encontrado em levantamento realizado com indivíduos de origem japonesa, com idade entre 20 a 39 anos e em país com melhor nível socioeconômico. Este estudo detectou um percentual de $3,1 \%$ para uma perda de 4 a 6 dentes $^{(19)}$. Aspectos metodológicos e tipo de população podem explicar as diferenças entre os dois estudos.

Observou-se na presente investigação uma associação significante entre o tabagismo e a perda dentária. Outros estudos também demonstraram essa associação(12,19-22). Recentemente, uma pesquisa de base populacional, realizada nos Estados Unidos da América, com mulheres pós-menopausadas, mostrou associação do hábito tabagístico com perda dentária $(\mathrm{OR}=1,82$; IC95\% 1,10$3,00)^{(12)} \mathrm{e}$, portanto, semelhante a do presente estudo.

Outra pesquisa americana, também de base populacional com aplicação de questionários, avaliou 3552 indivíduos com idade superior a 20 anos quanto à saúde bucal e o hábito de fumar, observando uma associação entre o hábito tabagístico e a má qualidade da saúde bucal (OR 1,42; IC95\% 1,07-1,88) $)^{(13)}$.

A idade superior a 60 anos também é fator de risco para perda dentária. No último levantamento epidemiológico 
nacional, ocorrido no Brasil em 2010 $0^{(14)}$, a perda dentária média para a faixa etária foi de 7,3 dentes.

Ao estratificar a amostra em fumantes, ex-fumantes e não fumantes, observou-se, no atual estudo, um maior percentual de pacientes com perda dentária no grupo de fumantes quando comparado ao de não fumantes. Em consonância com este dado, um estudo com 165 trabalhadores da zona rural, no sul do Brasil, observou uma maior probabilidade de periodontite e consequente perda dentária entre os fumantes quando comparados aos não fumantes ${ }^{(11)}$. Outro estudo, de base populacional, evidenciou que os fumantes apresentam uma chance seis vezes maior de perda dentária quando comparados aos não fumantes ${ }^{(12)}$. Um estudo de coorte prospectivo, realizado no período de 1986 a 2002, mostrou também que os fumantes apresentavam uma chance maior para perda dentária que os não fumantes ${ }^{(15)}$.

Em relação à alteração na cor dos dentes, houve uma diferença entre os grupos fumantes, ex-fumantes e não fumantes na presente investigação. Esta alteração tambem foi observada por outros autores ${ }^{(7,15)}$. Um estudo conduzido na comunidade do Reino Unido encontrou percentual de $28 \%$ para descoloração dos dentes entre os fumantes e $15 \%$ entre os não fumantes ${ }^{(23)}$, sendo este resultado inferior ao do presente estudo. Isto provavelmente porque a população investigada no presente estudo apresenta características socio-demográficas diferentes da população do Reino Unido. Além disso, estudaram-se pacientes de base ambulatorial, portanto, uma população com maior morbidade.

No presente estudo utilizaram-se apenas questionários para avaliação da saúde bucal. Este fato, no entanto, não constitui uma limitação importante, pois as perguntas foram claras e utilizaram uma definição rigorosa para qualidade da saúde bucal. Outro estudo também considerou questionários adequados para avaliação da saúde bucal ${ }^{(24)}$.

O poder e tamanho amostral do atual estudo foram adequados para demonstrar uma associação entre tabagismo e perda dentária, assim como alteração na cor dos dentes. Tomou-se o cuidado de lidar com o confundimento através da análise de regressão logística controlando para outras variáveis que poderiam estar associadas à má qualidade da saúde bucal. Com esta análise, observou-se um odds ratio satisfatório com intervalo de confiança adequado.

Este é o primeiro estudo realizado no Brasil, com pacientes atendidos em ambulatório de clínica médica, que evidencia o impacto negativo do tabagismo na saúde bucal dos pacientes. Os resultados encontrados fundamentam a necessidade de alerta à população, por parte dos profissionais da saúde, quanto ao risco do tabagismo sobre a saúde bucal e necessidade de acompanhamento odontológico precoce.

\section{CONCLUSÃO}

$\mathrm{Na}$ amostra estudada, o tabagismo está associado à deterioração da saúde bucal, causando, pois, um impacto negativo sobre esta.

Os autores não apresentam conflito de interesse.

\section{REFERÊNCIAS}

1. World Health Organization - WHO. WHO Report on the Global Tobacco Epidemic [updated Dec 2009]. Disponível em: http://www.who.int/tobacco/mpower/ en/index.html.

2. Abdullah AS, Husten CG. Promotion of smoking cessation in developing countries: a framework for urgent public health interventions. Thorax. 2004;59(3):623-30.

3. Instituto Nacional de Câncer - INCA. Agência de notícias: Ministério da Saúde informações [acesso em 2012 Dez 2]. Disponível em: http://www.inca.gov.br/ tabagismo/frameset.asp?item=publicacoes\&link=indic e.htm.

4. International Association for Dental Research. Policy statements: oral diseases related to tobacco use [acesso em 2012 Dez2]. Disponível em: http://www.aadronline. org/i4a/pages/index.cfm?pageid=3465\#TOBACCO.

5. Centers for Disease Control and Prevention (US). How Tobacco Smoke Causes Disease: The Biology and Behavioral Basis for Smoking-Attributable Disease: A Report of the Surgeon General [acesso em 2012 Dez 2]. Atlanta: Centers for Disease Control and Prevention; 2010. Disponível em: http://www.ncbi.nlm.nih.gov/ books/NBK53017/.

6. Lee YC, Marron M, Benhamou S. Active and involuntary tobacco smoking and upper aerodigestive tract cancer risks in a multicenter case-control study. Cancer Epidemiol Biomarkers Prev. 2009;18(12):335361.

7. Warnakulasuriya S, Dietrich T, Bornstein MM, Peidró EC, Preshaw PM, Walter C, et al. Oral health risks of tobacco use and effects of cessation. Int Dental J. 2010;60(1):7-30.

8. Tanaka K, Miyaka Y, Arakawa M. Sasaki S, Ohya Y. Household smoking and dental caries in schoolchildren: the Ryukyus Child Health Study. BMC Public Health. 2010;10(335):1-4.

9. Davis JM, Ramseier CA, Mattheos N, SchoonheimKlein M, Compton S, Al-Hazmi N, et al. Education 
of tobacco use prevention and cessation for dental professionals - a paradigm shift. Int Dental J. 2010;60(1):60-72.

10. Victoroff KZ, Dankulich-Huryn T, Haque S. Attitudes of incoming dental students toward tobacco cessation promotion in the dental setting. $\mathrm{J}$ Dent Educ. 2004;68(5):563-8.

11. Moimaz SA, Zina LG, Saliba O, Garbin CA. Smoking and periodontal disease: clinical evidence for an association. Oral Health Prev Dent. 2009;7(4):369-76.

12. Mai X, Wactawski-Wende J, Hovey KM, LaMonte MJ, Chen C, Tezal M, et al. Associations between smoking and tooth loss according to the reason for tooth loss: the Buffalo OsteoPerio Study. J Am Dent Assoc. 2013;144(3):252-65.

13. Liu Y. The relationship between lifestyle and selfreported oral health among American adults. Int Dental J. 2014;64(1):46-51.

14. Ministério da Saúde (BR). Projeto SBBrasil 2010: Pesquisa Nacional de Saúde Bucal [acesso em 2012 Dez 2]. Disponível em: dab.saude.gov.br/cnsb/sbbrasil/ index.html.

15. Dietrich T, Maserejian NN, Joshipura KJ, Krall EA, Garcia RI. Tobacco use and incidence of tooth loss among US male health professionals. J Dent Res. 2007;86(4):373-7.

16. World Health Organization - WHO. WHO Report on the Global Tobacco Epidemic, 2013. Enforcing bans on tobacco advertising, promotion and sponsorship [acesso em 2012 Dez 2]. Disponível em: http://www. who.int/tobacco/global_report/2013/en/.

17. World Health Organization, Department of Mental Health and Substance Dependence - Noncommunicable Diseases and Mental Health Cluster. International guide for monitoring alcohol consumption and related harm [acesso em 2012 Dez 2]. Geneva: WHO; 2000. Disponível em: http://whqlibdoc.who.int/hq/2000/ who_msd_msb_00.4.pdf.

18. American Dental Association. Brushing Your Teeth. [acesso em 2012 Dez 2]. Disponível em: http://www. mouthhealthy.org/en/az-topics/b/brushing-your-teeth.

19. Ojima M, Hanioka T, Tanaka K, Aoyama H. Cigarette smoking and tooth loss experience among young adults: a national record linkage study. BMC Public Health. 2007 [acesso em 2012 Dez 2];7:313-20. Disponível em: http://www.biomedcentral.com/1471-2458/7/313.

20. Piassi EO, Lara PA Fonseca DC, Fagundes VV. O Fumo como Fator Modificador da Doença Periodontal. Rev Intern de Periodontia Clínica. 2005;2(5):67-73.

21. Carvalho AE, Santos IG, Cury VF. A Influência do Tabagismo na Doença Periodontal: Revisão de Literatura. SOTAU Revista Virtual Odontol. 2008;2(5):7-12.

22. Sallum AW, César Neto JB, Sallum EJ. Tabagismo e a Doença Periodontal. Periodontia. 2007;17(2):45-53.

23. Alkhatib N, Holt RD, Bedi R. Smoking and tooth discolouration: findings from a national cross-sectional study. BMC Public Health. 2005;5(27):1-4.

24. Cascaes AM, Peres KG, Peres MA. Periodontal disease is associated with poor self-rated oral health among Brazilian adults. J Clin Periodontol. 2009;36(1):25-33.

\section{Endereço para correspondência:}

Eanes Delgado Barros Pereira

Departamento de Medicina Clínica (UFC)

Rua Professor Costa Mendes, 1608/4 andar

Bairro: Rodolfo Teófilo

CEP: 60430-140 - Fortaleza - CE - Brasil

E-mail: eanes@fortalnet.com.br 